\title{
Ipilimumab: A tale of twists and turns
}

\section{Dhiraj Abhyankar}

\section{Summary}

Today, ipilimumab has become a milestone in the management of cancer. Apart from being the first in new class of drug, it has redefined the way efficacy is evaluated and gave different classes of side effect profile. The journey till it became milestone was full of twists and turns. The man who discovered could had been a physician in traditional term, preferred working on what prevents attack of T-cell on cancer cells rather than "in thing" of those days of priming T-cells to attack cancer, and in spite of fantastic results in animal model, no major pharma companies were ready to pick up this drug because of patent mess.

Dr. James Allison is credited for the discovery of ipilimumab. He was a son of a doctor, and unlike his father, he was keen to get into a profession where the burden of being right every time is less and hence he preferred to be a scientist. So as to avoid going to the Vietnam War, he moved into research in life sciences. No wonder a harmonica player who finds gratification in blues music over symphony preferred to understand the hurdles in the attack of T-cells on cancer rather than working on the emerging field of immune attack on cancer cell itself. He never intended to develop anticancer drug but was exploring the "work ethics" of T-cells in relation to cancer. In the early days of his career, he was so much inspired by a seminar that he changed his line of research. He was convinced to lead the science of immunology in certain direction, but the current position would not have allowed him to work on that path hence he took sabbatical. In this period, he developed the structure of a gene and cloned it for T-cell antigen receptor. That was the beginning which ended up becoming ipilimumab. When most of the discoveries related to T-cells were directed toward pathways activating T-cells, Allison and others discovered something which prevents the activation of T-cells, the so-called "breaks" or checkpoint inhibitor.

There are two important stimulatory receptors on T-cells such as CD28 and cytotoxic T-lymphocyte-associated antigen 4 (CTLA-4) which attach on antigen presenting cell at ligand B7. CD28 upon engagement with B7 stimulates T-cells, whereas engagement of CTLA-4 to B7 antagonizes T-cell activation. This particular pathway prevents continuous stimulation of T-cells. It was also discovered that cancer cells hide from destruction from by T-cells by this pathway. Hence, it was hypothesized that blocking CTLA-4 would block the breaks of T-cells (i.e., increase the T-cell attack on cancer). ${ }^{[1]}$

Like any other drug discovery, once target got discovered, a drug (monoclonal antibody - anti CTLA-4) was developed against it. This was a unique drug which was blocking the breaks. That means the blockage of the target is going to

\begin{tabular}{|l|}
\hline Access this article online \\
\hline Quick Response Code: \\
\\
Website: www.sajc.org \\
\hline DOI: $10.4103 /$ sajc.sajc_231_17 \\
\hline
\end{tabular}

Pharm-Olam International Private Limited, Raleigh,

North Carolina, USA

Correspondence to: Dr. Dhiraj Abhyankar,

E-mail: dhiraj.abhyankar@pharm-olam.com unleash the T-cells against the tumor, but in the absence of T-cells, the blockage of target was not going to kill the cancer cells. The first experiment in mice has shown astonishing results. All mice in the control group have to be sacrificed, but all mice in the treatment group not only showed the complete response but also developed memory to fight subsequent challenge with the same tumor. This was unpreceded, a memory of immune system to fight cancer, just like infections. More than 300 peptides (vaccines) failed previously to elicit the response of T-cells against cancer cells because they were targeting more specific antigen on the cancer and was expecting T-cells to elicit response against cancer cells. But, because of breaks, T-cells were not able to attack it. This magic wand had potential not only to kill one tumor but also theoretically to cure all types of cancers. Many experiments proved in mouse model that this drug has potential to treat colon, renal, prostate, and breast cancers. Animal experiments and initial human experiments had proven that an adjuvant or immune response generating substances boosts the immune response when administered along with ipilimumab. However, in a landmark trial which gave approval for this drug, the response to adjuvant plus ipilimumab was equal to ipilimumab alone and now the drug is used without immune booster. ${ }^{[2]}$

There are many monoclonal antibodies which are chimeric (mouse + human); however, ipilimumab is developed in a unique way, i.e., the animal experiments were carried out on mouse origin antibody and human experiments were carried out on humanized antibody. Mechanism of action of the drug was such that if it would have been developed by a traditional route, i.e., evaluation of response on the basis of RECIST criteria, then it would have failed miserably. As in the initial period because of the infiltration of T-cells in the tumor cells, radiologically, the tumor increases in size, which can be termed as a progressive disease by RECIST and the patient would have been off trial. Hence, a biologist like Dr. Allison while monitoring the progress, closely allowed the study to progress till he could evaluate the disease-free survival and about $25 \%$ of patients showed long-term response in metastatic melanoma. Such response was never observed in this disease before. When the ipilimumab was going through the clinical trials, Pfizer was conducting studies on tremelimumab. Phase I and II data showed some response in metastatic melanoma, but Phase III data did not meet the primary objective. Many experts believe that the failure to meet primary objective in Phase III by tremelimumab was related to the way response was measured by the conventional RECIST criteria. This inability of RECIST criteria to evaluate the response in such immunotherapies leads to the frequent use of new response guideline known as Immune-Related Response Criteria. With

This is an open access journal, and articles are distributed under the terms of the Creative Commons Attribution-NonCommercial-ShareAlike 4.0 License, which allows others to remix, tweak, and build upon the work non-commercially, as long as appropriate credit is given and the new creations are licensed under the identical terms.

For reprints contact: reprints@medknow.com

How to cite this article: Abhyankar D. Ipilimumab: A tale of twists and turns. South Asian J Cancer 2018;7:193-4. 
its unique mechanism of action, distinct response patterns were observed and considered to be efficacy of the drug. Approximately $30 \%$ of patients treated with ipilimumab had disease control according to traditional RECIST criteria. They either had complete, partial response or stable disease. Some patients initially showed stable disease and then showed a decrease in tumor mass. The most interesting finding was that there was an initial increase in tumor mass and/or appearance of new lesions followed by decrease in the tumor mass or the tumor stopped growing further (stable disease). ${ }^{[3]}$

Ipilimumab not only revised the way response is evaluated, but also showed unique emergent side effects because of its unique mechanism of action. The new set of guidelines named as immune-related adverse events (irAEs) was introduced. These side effects included hypophysitis, colitis, hepatitis, pneumonitis, and rash. There are increasing case reports of patients who develop irAEs resembling inflammatory and rheumatic diseases such as arthritis, nephritis, myositis, and polymyalgia-like syndromes, and even type I diabetes in adults. ${ }^{[4]}$

No wonder that such a landmark drug had many twists in the patents which made it one of the most twisted affairs. The University of California, Berkeley, had experience to take the research and patents till preclinical stage only, but they patented clinical application of the invention in 1995 and moved ahead with licensing it out to a small company NeXstar Pharmaceuticals, which later on got merged with the biopharmaceutical company, Gilead Sciences Inc. Gilead sublicensed the rights to Medarex in 1999. Bristol-Myers Squibb (BMS) acquired Medarex in 2009. But, during this period, Medarex and Pfizer had signed an agreement in 2004 for co-development in spite of the fact that Pfizer was already into the development of its own anti-CTLA CP-675,206 (later named as tremelimumab). However, this collaboration lasted for a short duration, and finally, the product landed with BMS in 2005, who developed it further till commercialization. BMS had patented CTLA as a stimulator of T-cells and this prevented Dr. Allison to find a suitable developer for this drug before Medarex stepped in, as the patent position of this drug was considered "dirty" by potential investors.

Financial support and sponsorship

Nil.

Conflicts of interest

There are no conflicts of interest.

\section{References}

1. Leach DR, Krummel MF, Allison JP. Enhancement of antitumor immunity by CTLA-4 blockade. Science 1996;271:1734-6.

2. Hodi FS, O'Day SJ, McDermott DF, Weber RW, Sosman JA, Haanen JB, et al. Improved survival with ipilimumab in patients with metastatic melanoma. N Engl J Med 2010;363:711-23.

3. Wolchok JD, Hoos A, O'Day S, Weber JS, Hamid O, Lebbé C, et al. Guidelines for the evaluation of immune therapy activity in solid tumors: Immune-related response criteria. Clin Cancer Res 2009; 15:7412-20.

4. Michot JM, Bigenwald C, Champiat S, Collins M, Carbonnel F, Postel-Vinay S, et al. Immune-related adverse events with immune checkpoint blockade: A comprehensive review. Eur J Cancer 2016;54:139-48. 